\title{
Mapping of QTLs for floating ability in rice
}

\author{
Ritsuko Kawano $^{1,2)}$, Kazuyuki Doi ${ }^{1)}$, Hideshi Yasui ${ }^{1)}$, Toshihiro Mochizuki ${ }^{3)}$ and Atsushi Yoshimura*1) \\ 1) Plant Breeding Laboratory, Kyushu University, 6-10-1 Hakozaki, Higashi, Fukuoka 812-8581, Japan \\ 2) Japan Society for the Promotion of Science, 8 Ichibancho, Chiyoda, Tokyo 102-8472, Japan \\ 3) Laboratory of Agricultural Ecology, Kyushu University, 111 Harumachi, Kasuya-machi, Kasuya, Fukuoka 811-2307, Japan
}

\begin{abstract}
Deepwater rice (floating rice) can survive under flooded conditions because of their floating ability. We conducted genetic analysis to elucidate the genetic control of floating ability by using an $\mathrm{F}_{2}$ and $\mathrm{BC}_{3} \mathrm{~F}_{2}$ populations derived from a cross between deepwater and non-deepwater rice varieties. Internode elongation is the most important trait responsible for this adaptation, and is characterized by two factors: timing of the initiation of elongation and the rate of elongation. The position of the lowest elongated internode (LEI) and the rate of internode elongation (RIE) were used to measure floating ability. Two QTLs for LEI were detected on chromosomes 3 (qLEI3) and 12 (qLEI12). For RIE, two QTLs were detected on chromosomes 1 (qRIEI) and 12 (qRIE12). We confirmed the genetic effects and map positions of $q L E I 3, q L E I 12$ and $q$ RIE12 by using $\mathrm{BC}_{3} \mathrm{~F}_{2}$ populations. Characterization of near-isogenic lines of $q L E I 3, q L E I 12$ and $q R I E 12$ revealed that the LEI and RIE are at least partly controlled by different genetic pathways. Observation of near-isogenic lines suggested that the introgressed segment of qLEI12 and qRIE12 of chromosome 12 affected the deepwaterresponsive elongation.
\end{abstract}

Key Words: deepwater rice, QTL, lowest elongated internode, rate of internode elongation.

\section{Introduction}

Deepwater rice (floating rice) is cultivated in floodprone areas of South and Southeast Asia. In these areas, they are able to survive periods during the rainy season when the water level gradually rises to a depth of $1 \mathrm{~m}$ or more. By rapidly elongating their culms, the plants can avoid complete submergence and thereby survive the flooding. Plant length equals the sum of the lengths of the internodes, leaf sheaths and blades. In particular, changes in the internode length contribute most to the rapid elongation under rising water levels (Vergara et al. 1976). Deepwater rice is useful for the maintenance of plant diversity and of traditional ecosystems, and for stabilizing food production in flooded wetlands (Islam and Bennett 1998). In addition, the plant is a good material for studying stem elongation (Kende et al. 1998) and the genetic bases of phenotypic plasticity (Eiguchi et al. 1993).

Floating ability of rice, defined as survival ability under gradually increased rising water levels, involves two factors (Inouye 1987). One is the period when the plants start their internode elongation. Deepwater rice varieties initiate internode elongation early in the vegetative stage as compared with non-deepwater rice varieties. Even deepwater rice would die if it were submerged before it could begin internode elongation (Vergara et al. 1975). The position of the lowest elongated internode (LEI) is an appropriate indicator

Communicated by M. Yano

Received October 22, 2007. Accepted December 13, 2007.

*Corresponding author (e-mail: ayoshi@agr.kyushu-u.ac.jp) for measuring this characteristic (Inouye and Mogami 1980). The second factor is the rate of internode elongation. If rice plants can elongate their internodes rapidly, this response is advantageous and favors survival during flooding. The rate of internode elongation (RIE) has been defined to evaluate this factor (Takahashi and Mochizuki 2000). Therefore, both LEI and RIE are critical for rice survival in flooded areas (Inouye 1987).

Quantitative trait loci (QTL) analysis and subsequent dissection of each QTL in uniform genetic backgrounds enable us to clearly characterize genetical and physiological evidences of complex traits. This genetical approach can be easily achieved by the use of abundant DNA markers in rice. Using the plant materials such as $\mathrm{F}_{2}$, advanced backcross progeny and near-isogenic lines (NILs), we analyzed two important factors affecting floating ability, LEI and RIE, in the present study.

\section{Materials and Methods}

\section{Plant materials}

An $F_{2}$ population consisting of 116 plants derived from a cross between 'Taichung 65 (T65)' and 'Bhadua' (Oryza sativa $\mathrm{L}$.) was used for the QTL analysis. Bhadua is an Indica variety that originated in Bangladesh, and is a typical deepwater rice cultivar. T65, a Japonica variety from Taiwan, is a non-deepwater rice.

To further map QTLs in the T65 background, $\mathrm{BC}_{3} \mathrm{~F}_{2}$ populations were generated. Seventy-five $\mathrm{BC}_{3} \mathrm{~F}_{1}$ plants were obtained by three backcrosses to the $F_{1}$ plants with $\mathrm{T} 65$. 
Because QTLs were detected on chromosomes 1, 3 and 12 in $\mathrm{F}_{2}$ analysis, we conducted marker assisted selection in the $\mathrm{BC}_{3} \mathrm{~F}_{1}$ by using simple sequence repeat (SSR) markers (Fig. 1) defining these QTL regions on chromosomes 1, 3 and 12. One plant heterozygous at the markers of chromosome 3 was selected. The result of whole genome survey using $125 \mathrm{SSR}$ markers revealed that the $\mathrm{BC}_{3} \mathrm{~F}_{1}$ plant still retained the retained two regions (RM3627-RM7075 and RM1003) on chromosome 1, one region (RM6997RM8217) on chromosome 4 and one region (RM3155) on chromosome 8 (Fig. 1). The self-pollinated progeny of the $\mathrm{BC}_{3} \mathrm{~F}_{1}$ plant was referred to as $\mathrm{BC}_{3} \mathrm{~F}_{2}-\mathrm{A}$ and used for QTL analysis. Similarly, another $\mathrm{BC}_{3} \mathrm{~F}_{1}$ plant that was heterozygous for the markers of chromosome 12 was selected to obtain the $\mathrm{BC}_{3} \mathrm{~F}_{2}-\mathrm{B}$ population. The $\mathrm{BC}_{3} \mathrm{~F}_{1}$ plant retained three regions on chromosomes 3 (RM3280), 4 (RM3524RM8217) and 7 (RM8261-RM1306) (Fig. 1). No $\mathrm{BC}_{3} \mathrm{~F}_{1}$
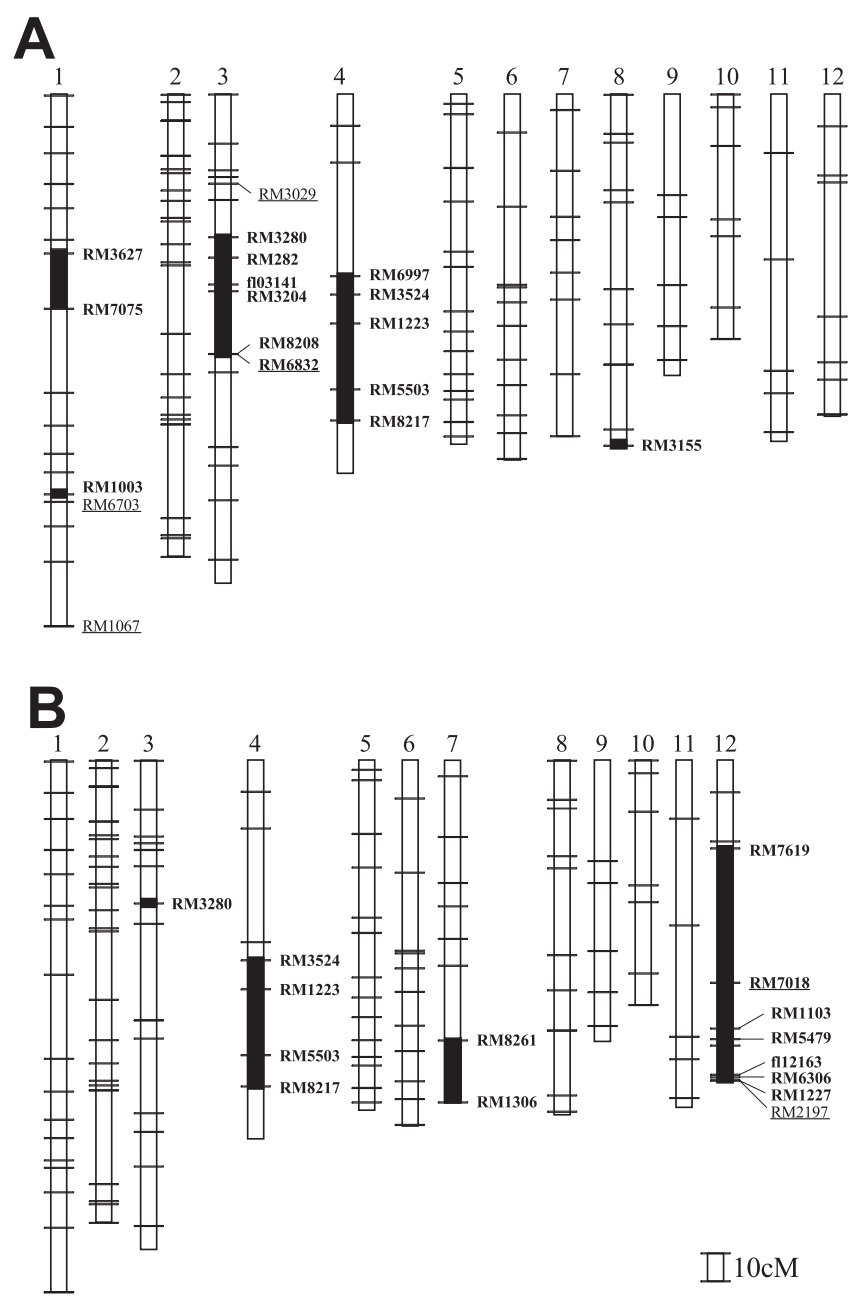

Fig. 1. Graphical genotypes of the selected $\mathrm{BC}_{3} \mathrm{~F}_{1}$ plants for $\mathrm{BC}_{3} \mathrm{~F}_{2}-\mathrm{A}$ (A) and $\mathrm{BC}_{3} \mathrm{~F}_{2}-\mathrm{B}$ (B) used for QTL analysis in the $\mathrm{BC}_{3} \mathrm{~F}_{2}$ and NIL development. Crossed lines show the position of SSR markers used for whole genome survey. The SSR markers used for the QTL analysis in $\mathrm{BC}_{3} \mathrm{~F}_{2}$ are shown in gothic. The SSR markers on chromosomes 1,3 and 12 used in the initial selection of $\mathrm{BC}_{3} \mathrm{~F}_{1}$ plants are underlined. plant for individual that was heterozygous at the region between RM6703 and RM1067 (chromosome 1) could be obtained. The $\mathrm{BC}_{3} \mathrm{~F}_{2}-\mathrm{A}$ and $\mathrm{BC}_{3} \mathrm{~F}_{2}-\mathrm{B}$ populations consisted of 93 and 97 plants, respectively.

Two NILs, NIL3 and NIL12, were selected from $\mathrm{BC}_{3} \mathrm{~F}_{2}-\mathrm{A}$ and $\mathrm{BC}_{3} \mathrm{~F}_{2}-\mathrm{B}$, respectively. NIL3 was fixed with Bhadua allele in the QTL region on chromosome 3. In NIL3, the other regions retained on chromosome 4 were eliminated but the two regions on chromosome 1 and one on chromosome 8 were still retained in heterozygous conditions. NIL12 was the NIL for the QTLs on chromosome 12 and still retained the other three regions on chromosomes 3,4 and 7 in heterozygous conditions.

\section{Evaluation of floating ability}

In 2001 , the $\mathrm{T} 65 \times$ Bhadua $\mathrm{F}_{2}$ seeds were germinated at $30^{\circ} \mathrm{C}$ in the dark for $48 \mathrm{~h}$. Each germinated seed was sown in a $1.5 \mathrm{~L}$ plastic pot $(11.3 \mathrm{~cm}$ diameter $\times 13.0 \mathrm{~cm}$ depth $)$ containing $1.7 \mathrm{~kg}$ paddy soil fertilized with $0.5 \mathrm{~g} \mathrm{~N}, 0.5 \mathrm{~g} \mathrm{P}_{2} \mathrm{O}_{5}$, and $0.5 \mathrm{~g} \mathrm{~K}_{2} \mathrm{O}$. For determining LEI, we monitored the leaf number on the main culm from the 5 th leaf stage by marking leaves with oil-based colored ink. When the plants reached the 7 th leaf stage, they were partly submerged to a depth of $10 \mathrm{~cm}$ above the soil surface in a $300 \mathrm{~L}$ plastic tank $(80 \mathrm{~cm}$ diameter $\times 60 \mathrm{~cm}$ depth). When internode elongation was observed, we recorded the LEI of each plant. The internode position corresponded to the leaf number, for instance, the internode whose upper node joining the 7 th leaf was designated as the 7th internode.

Subsequently, plants that had begun internode elongation were subjected to rising water levels so that we could measure RIE. Each pot was suspended in water in a $2 \mathrm{~m}$-deep concrete tank using a $2.5 \mathrm{~m}$ rope. The water depth was adjusted daily by lengthening the rope to lower each pot further beneath the water (i.e., each plant was lowered at a different rate). Positions of the plants were adjusted to ensure that half of the uppermost fully expanded leaf blade on the main culm in each plant remained above the water. Each individual was treated independently for 3 weeks. If a plant reached the bottom of the tank in less than 3 weeks, the treatment was ended. Immediately before and after the rising water treatment, we measured the lengths of all elongated internodes of the main culm. The lengths of the internodes that elongated during the treatment were given by subtraction, and RIE $(\mathrm{cm}$ $\mathrm{d}^{-1}$ ) was calculated on the basis of this change in length. Any internode longer than $1 \mathrm{~cm}$ was designated as an elongated internode (Takahashi et al. 1988). The experiment was performed under a $16 \mathrm{~h}$ photoperiod, with natural light supplemented with light from sodium lamps to prevent the occurrence of internode elongation accompanying with the initiation of the flower buds. The experiments using the $\mathrm{BC}_{3} \mathrm{~F}_{2}$ populations and NILs were conducted using the method basically the same as described above. $\mathrm{BC}_{3} \mathrm{~F}_{2}-\mathrm{A}$ was evaluated for LEI, but both LEI and RIE were measured in $\mathrm{BC}_{3} \mathrm{~F}_{2}-\mathrm{B}$ in 2003. In 2004, NILs were assayed for both traits. 
Genotype identification using DNA markers

Ninety restriction fragment length polymorphism (RFLP) and 30 SSR markers (Fig.3) were used for the whole genome genotype identification of the $F_{2}$ population derived from a cross T65/Bhadua. Total 125 SSR markers (Fig. 1) were used for marker assisted selection and QTL analysis in $\mathrm{BC}_{3} \mathrm{~F}_{1}$ and $\mathrm{BC}_{3} \mathrm{~F}_{2}$. Probes for the RFLP markers included Nipponbare genomic clones (Npb; Saito et al. 1991), RFLP clones from Japan's Rice Genome Project (Harushima et al. 1998), and Ky11, a genomic clone of IR24, an Indica variety (Tsunematsu et al. 1996). The Npb clone was designated as XNpb on the linkage map. DNA extraction and Southern hybridization analysis were conducted following Kurata et al. (1994) for determination of the genotype of the RFLP loci. For the $\mathrm{BC}_{3}$ materials, we performed DNA extraction according to the method of Dellaporta et al. (1983) with minor modifications. The information on the SSR markers (RM numbers) was obtained from McCouch et al. (2002). SSR markers with the prefix "fl" were developed by the authors. For the f103141 marker, we used the forward primer 5'-GAGGAAGGAGACGCAGCA-3' and the reverse primer 5'-CACCGGGCTCCAGTTTTC-3'. The primer set 5'-CGTCTGCATCGGAGTAGTAGC-3' and 5'-CACCAC CCATAGCCATAGCC-3' was used for fl12163. These primers were designed from the genomic sequence of the rice (GenBank accessions AC137597 and AL713940). The PCR was conducted using the $15 \mu$ l of PCR reaction mixture: $1 \times$ PCR buffer, $1.5 \mathrm{mM} \mathrm{MgCl}_{2}, 0.2 \mathrm{mM}$ dNTPs, $0.15 \mu \mathrm{l}$ of 5 unit Taq DNA polymerase and $1 \times$ PCR dye $(0.015 \%$ bromophenolblue and 3\% glycerol) and 0.27 pmol primers, with $5 \mathrm{ng}$ DNA templates. The conditions for amplification were 35 cycles of denaturation at $94^{\circ} \mathrm{C}$, annealing at $55^{\circ} \mathrm{C}$, and extension at $72^{\circ} \mathrm{C}$ for $30 \mathrm{~s}$, respectively. For genotype identification, the PCR products were electrophoresed on $4 \%$ agarose gel.

\section{Linkage map construction and QTL analysis}

Linkage analysis was conducted with MAPMAKER/ Exp v. 3.0 (Lander et al. 1987). The QTL analysis was performed with Windows QTL Cartographer Version 2.0 (Basten et al. 2004). Both simple interval mapping (SIM) and composite interval mapping (CIM) were used to detect QTLs. QTLs for LEI and RIE were declared significant at $P<0.01$, with the LOD score given by 1000 permutations (Churchill and Doerge 1994, Doerge and Churchill 1996).

\section{Results}

\section{QTL analysis in $F_{2}$ population}

Bhadua, a typical deepwater rice variety, started internodal elongation at early vegetative stage, and the LEI value was $6.4 \pm 0.5$. The non-deepwater rice variety parent, T65, showed $13.7 \pm 0.5$ of LEI. Average of the RIE of Bhadua and T65 were $8.1 \mathrm{~cm} \mathrm{~d}^{-1}$ and $1.5 \mathrm{~cm} \mathrm{~d}^{-1}$, respectively. The two parameters, LEI and RIE, reflected the fact that Bhadua had higher floating ability than that of T65.
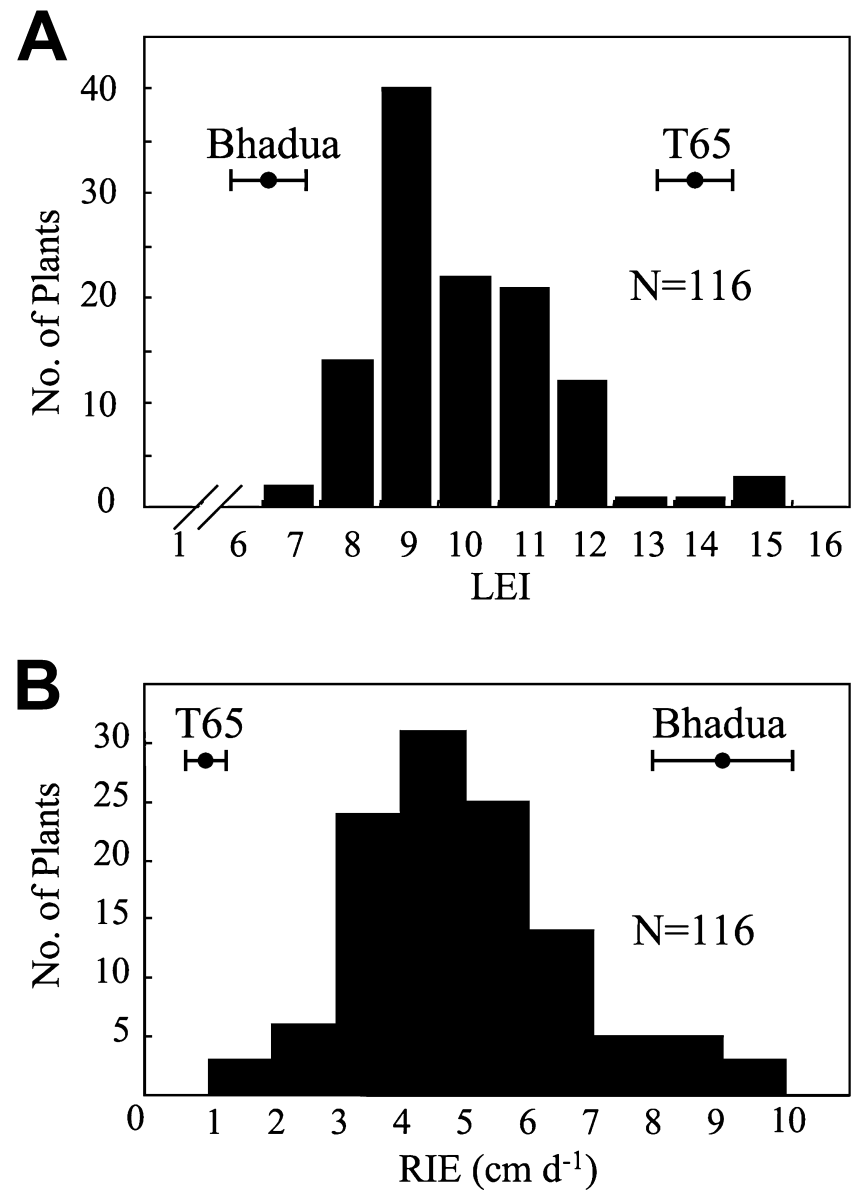

Fig. 2. Frequency distributions of (A) LEI and (B) RIE in the $F_{2}$ population $(\mathrm{T} 65 \times$ Bhadua). Points and ranges indicate the average and standard deviation, respectively. LEI were $13.7 \pm 0.5$ for T65 and $6.4 \pm 0.5$ for Bhadua, respectively. RIE were $1.6 \pm 0.2$ and $8.1 \pm 1.0$ for T65 and Bhadua, respectively.

The $\mathrm{F}_{2}$ population showed a continuous frequency distribution of LEI (Fig. 2A). Three plants showed LEI of 15, which was higher than the average of $\mathrm{T} 65$, but no plant showing an LEI value lower than that of Bhadua. Considering the environmental errors, it was not regarded as transgressive segregation. The frequency distribution of RIE in the $F_{2}$ population was also continuous (Fig. $2 B$ ). RIE ranged from 1.0 to $9.2 \mathrm{~cm} \mathrm{~d}^{-1}$, and transgressive segregation was not observed. The correlation between LEI and RIE was significant at $P<0.01(\mathrm{r}=-0.61)$.

The linkage map contained 120 DNA markers that covered the total genome, with an average interval of $16.5 \mathrm{cM}$ between markers (Fig. 3). Threshold values of LOD score given by 1000 permutations were 4.6 for LEI and 4.2 for RIE $(P<0.01)$. Two QTLs for LEI were detected on chromosomes 3 (qLEI3) and 12 (qLEI12) (Fig. 3). For RIE, two QTLs were detected on chromosomes 1 and 12. On the regions of $q L E I 3, q L E I 12, q R I E 1$ and $q R I E 12$, LOD scores peaked in the marker intervals XNpb144-RM8208, RM5479-fl12163, XNpb113-G54 and RM5479-fl12163, respectively (Fig. 3 and Table 1). The Bhadua allele lowered 

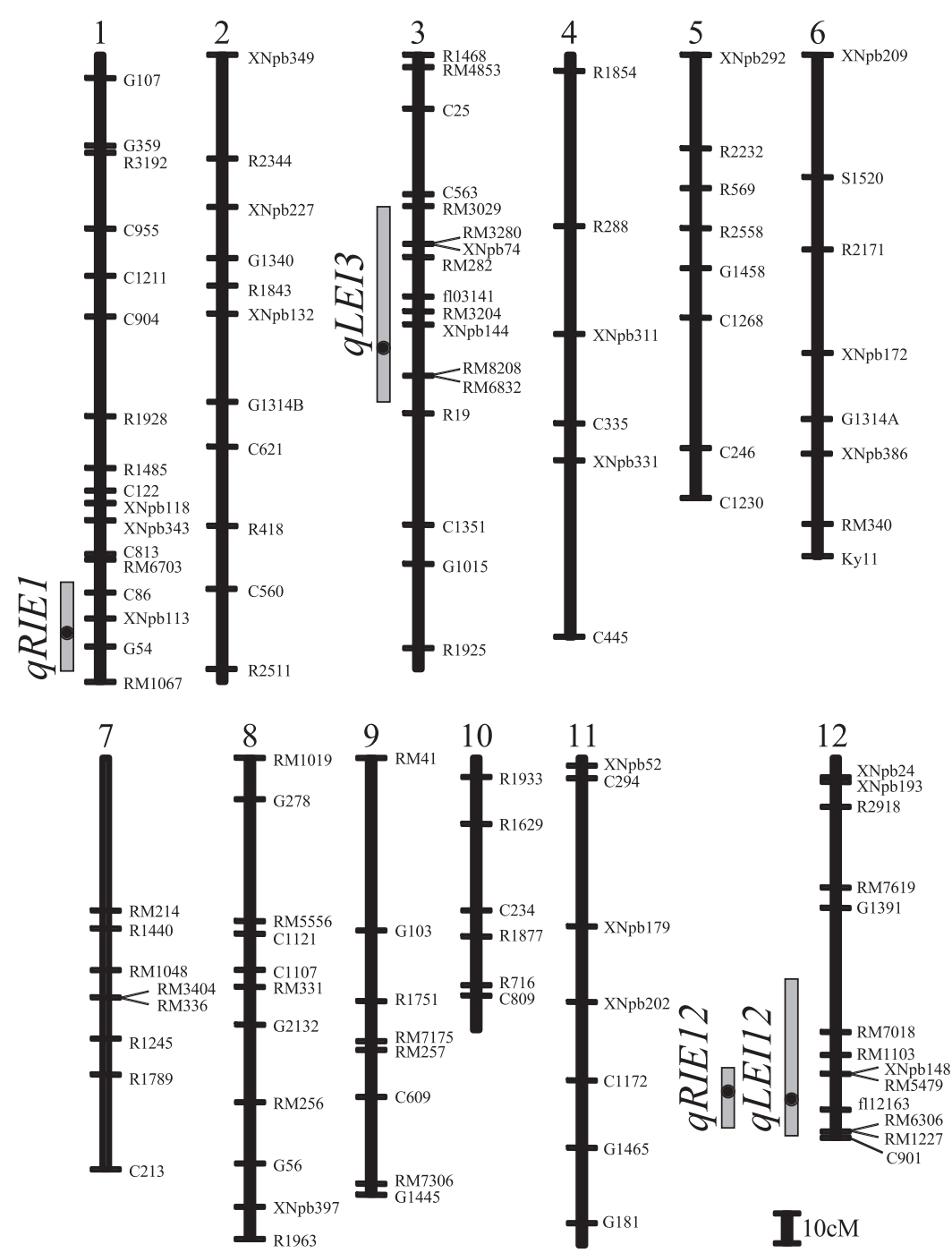

Fig. 3. A linkage map of the $\mathrm{F}_{2}$ population (T65 $\times$ Bhadua). The vertical bars to the left of the maps indicate the regions in which the LOD scores are significant for the trait at $1 \%$ level. The filled circles on the bars indicate the position of the peak LOD score for each QTL.

Table 1. Characteristics of the QTLs that affect LEI and RIE detected in the T65/Bhadua $F_{2}$ population

\begin{tabular}{clclccccccc}
\hline \hline Trait & QTL & Chromosome & Marker interval & Pos. ${ }^{a}$ & Threshold & LOD score & $\mathrm{r}^{2}(\%)^{c}$ & $\begin{array}{c}\text { Additive } \\
\text { effect }^{d}\end{array}$ & $\begin{array}{c}\text { Dominance } \\
\text { effect }^{e}\end{array}$ \\
\hline LEI & $q$ LEI3 & 3 & XNpb144-RM8208 & 6.0 & \multirow{2}{*}{4.6} & 9.0 & 40.7 & -1.5 & -0.8 \\
& qLEI12 & 12 & RM5479-f112163 & 10.0 & & 11.7 & 38.5 & -1.2 & -0.6 \\
\hline \multirow{2}{*}{ RIE } & qRIE1 & 1 & XNpb113-G54 & 4.0 & \multirow{2}{*}{4.2} & 7.5 & 30.6 & 2.3 & -0.5 \\
& qRIE12 & 12 & RM5479-f112163 & 6.0 & & 5.3 & 22.2 & 0.9 & 0.6 \\
\hline
\end{tabular}

${ }^{a}$ Genetic distance (cM) from the left marker of each interval where the peak LOD was scored.

${ }^{b}$ Given by 1000 permutations at $P<0.01$.

${ }^{c}$ Phenotypic variation explained by a single QTL.

${ }^{d}$ The values were calculated based on the following formula: ((trait values of Bhadua homozygotes) - (trait values of T65 homozygotes))/2.

${ }^{e}$ The values were calculated based on the following formula: (trait values of heterozygotes)-(mid-point values).

LEI, and increased RIE. By CIM technique, the peak for $q L E I 3$ appeared on the interval RM282-f103141. The interval was different from the result of SIM, but coincided with the results of the experiments using $\mathrm{BC}_{3} \mathrm{~F}_{2}$-A described below. On the other QTLs, the intervals where the peak LOD appeared were the same in SIM and CIM. Any additional
QTLs by CIM were not detected for neither LEI nor RIE.

Mapping and characterization of QTLs in the nearly isogenic background of T65

Fig. 4 shows the frequency distributions of LEI and $\mathrm{RIE}$ in $\mathrm{BC}_{3} \mathrm{~F}_{2}-\mathrm{A}$ and $\mathrm{BC}_{3} \mathrm{~F}_{2}-\mathrm{B}$. The LEI of both populations 

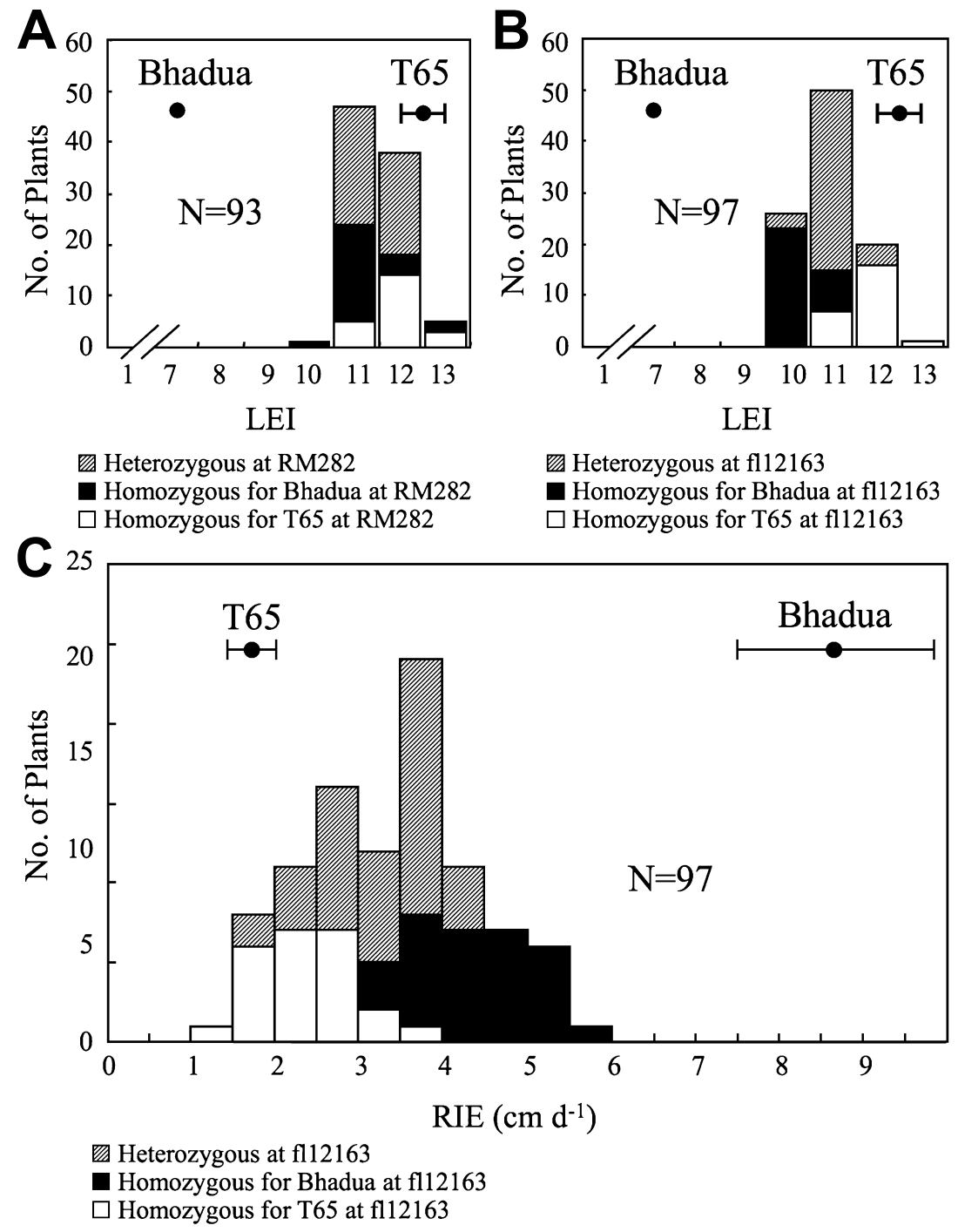

Fig. 4. Frequency distributions of $\mathrm{LEI}$ in $\mathrm{BC}_{3} \mathrm{~F}_{2}-\mathrm{A}(\mathrm{A})$ and $\mathrm{BC}_{3} \mathrm{~F}_{2}-\mathrm{B}(\mathrm{B})$, and that of $\mathrm{RIE}$ in $\mathrm{BC}_{3} \mathrm{~F}_{2}-\mathrm{B}(\mathrm{C})$. Bars are filled differently according to the genotypes at the SSR markers nearest to the QTLs (A: RM282, B and C: f112163).

fell within four LEI classes (10, 11, 12 and 13). However, no clear bimodal segregation was observed. It was also impossible to divide the segregation of RIE in $\mathrm{BC}_{3} \mathrm{~F}_{2}$-B into distinct classes. In addition to the small variation on LEI from 10 to 13 , it is considered that the fluctuation by environmental conditions caused continuous frequency distribution. In this experiment, performed in 2003, T65 showed an LEI value of $12.5 \pm 0.7$, which was lower than the result in 2001 (LEI $13.7 \pm 0.5$ ). This difference was probably due to differences in experimental conditions, especially temperature. The temperature in 2003 was lower than that in 2001 (data not shown).

Because discrete segregation was not observed in the $\mathrm{BC}_{3} \mathrm{~F}_{2}$ progeny, we conducted QTL analysis in these populations. In the interval between the markers RM282 and fl03141, peak LOD was scored for LEI in the $\mathrm{BC}_{3} \mathrm{~F}_{2}$-A population (Table 2). No QTL was detected in the other retained regions on chromosomes 1,4 and 8 (data not shown). In $\mathrm{BC}_{3} \mathrm{~F}_{2}-\mathrm{B}$, the LOD score peaked in the interval between markers RM5479 and fl12163 for both LEI and RIE (Table 2). In the other segregation regions on chromosomes 3, 4 and 7, no QTL was detected (data not shown).

Two NILs, NIL3 and NIL12, were selected from the $\mathrm{BC}_{3} \mathrm{~F}_{2}-\mathrm{A}$ and $\mathrm{BC}_{3} \mathrm{~F}_{2}-\mathrm{B}$, respectively. NIL3 and NIL12 were assayed for LEI and RIE and compared with T65 (Table 3 ). LEI of NIL3 and NIL12 were significantly lower than that of T65. RIE of NIL12 was significantly higher than that of T65. However, RIE of NIL3 was not significantly different from that of T65, indicating that $q L E I 3$ did not affect RIE. These results confirmed the QTL analysis in $\mathrm{F}_{2}$ except that the effect of $q L E I 12$ to LEI was greater than that of $q L E I 3$ (Table 1, Table 2 and Table 3).

\section{Discussion}

This study is the first genetic analysis for both LEI and RIE as indicators of floating ability in rice. In addition, we obtained NILs for $q L E I 3$ and $q L E I 12 / q R I E 12$. These lines 
Table 2. Characteristics of the QTLs that affect LEI and RIE detected in the $\mathrm{BC}_{3} \mathrm{~F}_{2}-\mathrm{A}$ and $-\mathrm{B}$ populations

\begin{tabular}{clclrrrrrrr}
\hline \hline Trait & QTL & Chromosome & Marker interval & Pos. & Threshold & LOD score & $\mathrm{r}^{2}(\%)^{c}$ & $\begin{array}{c}\text { Additive } \\
\text { effect }^{d}\end{array}$ & $\begin{array}{c}\text { Dominance } \\
\text { effect }^{e}\end{array}$ \\
\hline LEI & $q$ LEI3 & 3 & RM282-f103141 & 4.0 & 3.5 & 4.2 & 20.2 & -0.4 & -0.1 \\
& qLEI12 & 12 & RM5479-f112163 & 10.0 & 3.3 & 20.4 & 65.7 & -0.8 & -0.0 \\
\hline RIE & qRIE12 & 12 & RM5479-f112163 & 5.0 & 3.5 & 20.5 & 64.7 & 1.0 & 0.1 \\
\hline
\end{tabular}

${ }^{a}$ Genetic distance (cM) from the left marker of each interval where the peak LOD was scored.

${ }^{b}$ Given by 1000 permutations at $P<0.01$.

${ }^{c}$ Phenotypic variation explained by a single QTL.

${ }^{d}$ The values were calculated based on the following formula: ((trait values of Bhadua homozygotes) -(trait values of T65 homozygotes))/2.

${ }^{e}$ The values were calculated based on the following formula: (trait values of heterozygotes)-(mid-point values).

Table 3. LEI and RIE of NIL3, NIL12 and T65 (The values indicate the average \pm standard deviation)

\begin{tabular}{lll}
\hline \hline Line & LEI & RIE \\
\hline NIL3 & $12.4 \pm 0.7 \mathrm{~b}^{a}$ & $1.6 \pm 0.2 \mathrm{a}$ \\
NIL12 & $10.5 \pm 0.5 \mathrm{a}$ & $3.1 \pm 0.6 \mathrm{~b}$ \\
\hline T65 & $13.3 \pm 0.6 \mathrm{c}$ & $1.4 \pm 0.2 \mathrm{a}$ \\
\hline
\end{tabular}

${ }^{a}$ Different letters indicate significant difference at $5 \%$ level by Tukey-Kramer multiple comparison.

will be used in physiological bioassays to further characterize each QTL. This genetic methodology should always be used to dissect complex traits to simple Mendelian factors (Yano et al. 2001).

Our method for measuring RIE is capable of measuring the full potential elongation rate (Takahashi and Mochizuki 2000). By evaluating both LEI and RIE, we found that LEI and RIE are under different control to some extent, although a QTL region on chromosome 12 ( $q$ LEI12 and $q R I E 12)$ is shared by both traits. This linkage between $q L E I 12$ and qRIE 12 is the cause of the observed correlation between LEI and RIE. To characterize the QTLs, we subjected two NILs, NIL3 and NIL12, to the assays for LEI and RIE. As we expected from the QTL analysis in the $\mathrm{F}_{2}$ population, qLEI3 did not affect the RIE (Table 3 ). This evidence clearly indicated that LEI and RIE should be treated as different traits.

We observed that NIL12 elongate its plant length up to near $2 \mathrm{~m}$ at the end of the rising water level treatment. In the paddy field, however, plant length of NIL12 was about $1 \mathrm{~m}$ (data not shown). These results indicated that NIL12 has ability to change its plant length depending on the water level conditions. It is suggested that $q L E I 12 / q R I E 12$ are involved in the deepwater-responsive elongation. Hence, qLEI12/ qRIE12 might explain a major portion of the phenotypic plasticity generally observed in deepwater rice varieties.

Nemoto et al. (2004) reported the results of their QTL analysis of early elongation ability in deepwater rice variety 'Goai' by using LEI as the indicator. They identified QTLs for LEI on chromosomes 3 and 12, which were located at almost the same positions as in our results. On the same region of chromosome 12, they also detected QTL affecting LEI from different deepwater variety 'Habiganj AmanVIII' (Tang et al. 2005). These QTLs for LEI are probably widely distributed among deepwater rice varieties. One of the deepwater tolerance genes, $d w 3$, was identified by Eiguchi et al. (1993). Plants with $d w 3$ in the background of T65 had an LEI value of 10 . Moreover, in their study, the rice plants survived a water level increase of $10 \mathrm{~cm}$ every other day. From these results, we hypothesize that these plants they assessed have RIE comparable to that of the NIL12. Hence, it is possible to consider that $d w 3$ is one of the alleles at $q L E I 12$ and/or $q R I E 12$. In the almost the same region of $q R I E 1$, Sripongpangkul et al. (2000) detected the QTL controlling internode elongation in gradual water level rising conditions.

We are now developing the NIL of qRIE1 to comprehensively understand the floating ability in rice. An immediate follow-up experiment to the current study would logically focus on elucidating whether $q L E I 12$ and $q R I E 12$ were the same gene or not. A high-resolution linkage analysis of these loci is currently underway to address this question.

\section{Acknowledgements}

We greatly thank for support by Research Fellowship of the Japan Society for the Promotion of Science for Young Scientists. This work was supported by Grants-in-Aid for Scientific Research from the Ministry of Education, Culture, Sports, Science, and Technology to R.K., T.M. and A.Y.

\section{Literature Cited}

Basten,C.J., B.S.Weir and Z.B.Zeng (2004) Windows QTL Cartographer Version 2.0. North Carolina State University, USA. Available online: http://statgen.ncsu.edu/qtlcart/WQTLCart.htm.

Churchill,G.A. and R.W.Doerge (1994) Empirical Threshold values for quantitative trait mapping. Genetics 138: 963-971.

Dellaporta, S.L., J.Wood and J.B.Hicks (1983) A plant DNA minipreparation: Version II. Plant Mol. Biol. Rep. 1: 19-21.

Doerge,G.A. and R.W.Churchill (1996) Permutation tests for multiple loci affecting a quantitative character. Genetics 142: 285-294.

Eiguchi,M., R.Sano, H.Y.Hirano and Y.Sano (1993) Genetic and developmental bases for phenotypic plasticity in deepwater rice. J. Hered. 84: 201-205.

Harushima, Y., M.Yano, A.Shomura, M.Sato, T.Shimano, Y.Kuboki, T.Yamamoto, S.Y.Lin, B.A.Antonio, A.Parco et al. (1998) A high-density rice genetic linkage map with 2275 markers using a single $\mathrm{F}_{2}$ population. Genetics 148: 479-494. 
Inouye,J. (1987) On floating rice and its ecological traits in Southeast Asia. The Southeast Asian Studies 25: 51-61.

Inouye,J. and Y.Mogami (1980) On the position of the lowest elongated internode of floating rice originated in different countries. Jpn. J. Trop. Agr. 24: 13-17.

Islam,Q.R. and S.L.Bennett (1998) Role of deepwater rice (Oryza sativa $\mathrm{L}$.) to maintain aquatic environment in deeply flooded wetlands. In Proceedings of the 10th European Weed Research Society Symposium on Aquatic Weeds. Online version: http:// bicn.com/wei/resources/IslamBennett1998.htm.

Kende,H., E.van der Knaap and H.T.Cho (1998) Deepwater rice: A model plant to study stem elongation. Plant Physiol. 118: $1105-1110$.

Kurata,N., Y.Nagamura, K.Yamamoto, Y.Harushima, N.Sue, J.Wu, B.A.Antonio, A.Shomura, T.Shimizu, S.Y.Lin et al. (1994) A 300 kilobase interval genetic map of rice including 883 expressed sequences. Nat. Genet. 8: 365-372.

Lander,E.S., P.Green, J.Abrahamson, A.Barlow, M.J.Daly, S.E. Lincoln and L.Newbug (1987) Mapmaker: an interactive computer package for constructing primary genetic linkage maps of experimental and natural populations. Genomics 1: 174-181.

McCouch,S.R., L.Teytelman, Y.Xu, K.B.Lobos, K.Clare, M.Walton, B.Fu, R.Maghirang, Z.Li, Y.Xing et al. (2002) Development and mapping of 2240 new SSR markers for rice (Oryza sativa L.). DNA Res. 9: 199-207 (with supplement, p. 257-279).

Nemoto, K., Y.Ukai, D.Q.Tang and Y.Kasai (2004) Inheritance of early elongation ability in floating rice revealed by diallel and QTL analysis. Theor. Appl. Genet. 109: 42-47.

Saito, A., M. Yano, N. Kishimoto, M.Nakagahara, A. Yoshimura,
K.Saito, S.Kuhara, Y.Ukai, M.Kawase, T.Nagamine et al. (1991) Linkage map of restriction fragment length polymorphism loci in rice. Jpn. J. Breed. 41: 665-670.

Sripongpangkul,K., G.B.T.Posa, D.W.Senadhira, D.Brar, N.Huang, G.S.Khush and Z.K.Li (2000) Genes/QTLs affecting flood tolerance in rice. Theor. Appl. Genet. 101: 1074-1081.

Takahashi,T., A.M.Mazaredo and B.S.Vergara (1988) Expression of floating ability on non-floating ability. Jpn. J. Crop Sci. 57 (Extra issue 1): 113-114 (in Japanese with English summary).

Takahashi,U. and T.Mochizuki (2000) Varietal differences in internode elongation ability of deepwater rice. Jpn. J. Crop Sci. 69 (Extra issue 1): 198-199 (in Japanese with English summary).

Tang,D.Q., Y.Kasai, N.Miyamoto, Y.Ukai and K.Nemoto (2005) Comparison of QTLs for early elongation ability between two floating rice cultivars with a different phylogenetic origin. Breed. Sci. 55: 1-5.

Tsunematsu,H., A.Yoshimura, Y.Harushima, Y.Nagamura, N.Kurata, M.Yano, T.Sasaki and N.Iwata (1996) RFLP framework map using recombinant inbred lines in rice. Breed. Sci. 46: 279-284.

Vergara,B.S., A.Nazaredo, S.K.DeDatta and W.Abilay (1975) Plant age and internode elongation in floating rice varieties. In: Proceedings of the International Seminar on Deep-water Rice. p. 178-183.

Vergara,B.S., B.Jackson and S.K.DeDatta (1976) Deepwater rice and its response to deepwater stress. In: "Climate and Rice" IRRI (ed.), IRRI, Los Baños, Philippines. p. 301-319.

Yano,M., S.Kojima, Y.Takahashi, H.X.Lin and T.Sasaki (2001) Genetic control of flowering time in rice, a short-day plant. Plant Physiol. 127: 1425-1429. 